\title{
Mechanical energy input to the world oceans due to atmospheric loading
}

\author{
Wei Wang ${ }^{+}$, Cheng Chun Qian ${ }^{+}$, \& Rui Xin Huang*
}

+Physical Oceanography Laboratory, Ocean University of China, Qingdao 266003,

Shandong, P. R. China

*Department of Physical Oceanography, Woods Hole, MA 02543, USA

Manuscript accepted by Chinese Science Bulletin 


\begin{abstract}
Mechanical energy input to the oceans is one of the most important factors controlling the oceanic general circulation. The atmosphere transports mechanical energy to the oceans primarily through wind stress, plus changes of the sea level pressure (the so-called atmospheric loading). The rate of mechanical energy transfer into the ocean due to atmospheric loading is calculated, based on TOPEX/POSEIDON data over ten-year period (1993-2002). The rate of total energy input for the world oceans is estimated at $0.04 T W\left(1 T W=10^{12} W\right)$, and most of this energy input is concentrated in the Southern Oceans and the Storm Tracks in the Northern Hemisphere. This energy input varied greatly with time, and the amplitude of the interannual variability over the past ten years is about $15 \%$.
\end{abstract}




\section{Introduction}

Mechanical energy input from the atmosphere is one of the most important sources of energy driving the oceanic general circulation (Huang, 2004, Wunsch and Ferrari, 2004). This energy is transferred to the oceans, mostly through sea surface wind stress and atmospheric pressure perturbations, and it can be roughly separated into following categories: First, wind energy input through the surface waves, which is estimated as 60TW (Wang and Huang, 2004a). Second, wind energy input through the Ekman layer, which can be further separated into the near-inertial frequency range, 0.5 0.7TW (Alford 2003, Watanabe and Hibiya, 2002), and the sub-inertial frequency range, 2.4TW (Wang and Huang, 2004b). Third, wind energy input through the surface geostrophic current, 1.3TW (Wunsch, 1998). Fourth, the contribution due to atmospheric loading and this is the focus of this study.

Sea level atmospheric pressure varies with time and space, mostly due to the moving storms and other perturbations. Because of changes in sea level atmospheric pressure, water in the ocean moves in response, leading to changes in sea level. Such changes are relatively small and thus neglected in most oceanic circulation models. However, it is a vitally important correction term needed in processing satellite altimeter data. The oceanic response to atmospheric pressure perturbations involves complicated physical processes in the oceans, which has been the focus of many studies. For the most comprehensive review, the reader is referred to Wunsch and Stammer (1997).

Changes of sea level in the global oceans in response to atmospheric pressure perturbations are complicated and difficult to calculate. The "inverted barometer response” has been widely used in processing altimeter data (Fu and Pihos, 1994; Gaspar and Ponte, 1997). This approach is based on the assumption that sea level 
responses to the local sea level pressure (with a global correction) instantaneously. The errors introduced by this approach are small in general, except some special location, such as near the western boundaries, as discussed in previous studies (Wunsch and Stammer, 1997).

Our goal here is to estimate the global energy input to the oceans through atmospheric pressure perturbations. There are two possible approaches for this problem, i.e., using a numerical model or using observations. In order to avoid the uncertainty associated with numerical modeling our approach here is based on satellite data analysis, plus surface pressure data from data-assimilated models. In calculating the work done by atmospheric loading on the oceans, sea level pressure and the vertical velocity are needed. There are a variety of data of the global sea level pressure available (e.g., NCEP and ECMWF), while the vertical velocity at the sea surface is small and difficult to measure. In this study we use the TOPEX/POSEIDON altimeter data, which covers most parts of the world ocean with relatively high spatial resolutions and has a 10 -year long time series with a basic sampling rate of 10 days or shorter. In particular, measurements are repeated and the sampling rate is doubled at each crossover point of the TOPEX/POSEIDON altimeter.

\section{Formulation}

At any given position and time, the rate of mechanical energy input due to atmospheric loading is

$$
w(x, y, t)=-p(x, y, t) v(x, y, t)
$$

where $p(x, y, t)$ is the sea level atmospheric pressure, $v(x, y, t)=\frac{d \eta(x, y, t)}{d t}$ is the vertical velocity at the sea surface, $\eta$ is the sea level. Averaged over a period of $T$, the 
rate of energy input at a given position is

$$
\bar{w}(x, y)=<-p v>
$$

where $<>$ indicates the time average over the period $T$. If both the pressure and sea level are separated into the mean and perturbations

$$
p=p_{0}+p^{\prime}, \quad v=v_{0}+v^{\prime},
$$

then

$$
\bar{w}(x, y)=<-p_{0} v_{0}>+<-p_{0} v^{\prime}>+<-p^{\prime} v_{0}>+<-p^{\prime} v^{\prime}>.
$$

The first three terms on the right hand side of above equation vanish, so the mean energy input is

$$
\bar{w}(x, y)=<-p^{\prime} v^{\prime}>=-\frac{1}{T} \int_{0}^{T} p^{\prime} v^{\prime} d t=-\frac{1}{T} \int_{0}^{T} p^{\prime} \frac{d \eta^{\prime}}{d t} d t
$$

where $\eta^{\prime}$ is the sea level anomaly. Note that sea level anomaly can be induced by many dynamical processes, such as the atmospheric loading, wind stress, heating, precipitation/evaporation, and other dynamic processes; thus, pressure work obtained through altimeter data include contributions from all these processes.

The rate of total energy input into the world oceans is

$$
W=\iint \bar{w} d x d y=-\frac{1}{T} \iiint_{0}^{T} p^{\prime} \frac{d \eta^{\prime}}{d t} d t d x d y
$$

A major technical difficulty is the following: spatial and temporal resolution of sea level data from satellite altimeter is rather low. Sea level data at a given station is available roughly for every 10 days only, so this is the same order as the synoptical time scale for pressure. Consequently, two data samples of sea level $\eta^{\prime}$ at a given station may belong to different synoptical events. If $\frac{d \eta^{\prime}}{d t}$ calculated as the mean rate of sea level change over such a period is substituted in Eq.(1) to calculate the energy input, the 
results are unreliable. To overcome such a problem, Eq.(1) is rewritten by integration-by-part

$$
\bar{w}(x, y)=-\frac{1}{T} \int_{0}^{T} p^{\prime} \frac{d \eta^{\prime}}{d t} d t=-\frac{1}{T}\left(\left.p^{\prime} \eta^{\prime}\right|_{T}-\left.p^{\prime} \eta^{\prime}\right|_{0}-\int_{0}^{T} \eta^{\prime} \frac{d p^{\prime}}{d t} d t\right)
$$

Since sea level pressure is based on daily mean data, its time rate of change can be calculated accurately the NCEP data; meanwhile $\eta^{\prime}$ at each crossover point is based on the satellite altimeter data, without introducing any additional errors. Therefore, using Eq.(2) can produce more accurate energy input averaged over one day. For each satellite observation of $\eta^{\prime}$, there is a corresponding $w(x, y, t)$. If the sample number is large enough, the contribution of $-\frac{1}{T}\left(\left.p^{\prime} \eta^{\prime}\right|_{0} ^{T}\right)$ in Eq.(2) approaches zero, thus Eq.(2) is reduced to

$$
\bar{w}(x, y)=\frac{1}{T} \int_{0}^{T} \eta^{\prime} \frac{d p^{\prime}}{d t} d t
$$

\section{Results and discussion}

Our calculation is based on TOPEX/POSEIDON altimeter data provided by AVISO. Although data collected through all orbit cycles can be used in the calculation, there is a mismatch between the sampling frequency (about 10 days) and the frequency of the sea level atmospheric pressure perturbations (on the order of days). Although NCEP data provides daily mean pressure, sea level at a given station observed from satellite come in every 10 days. This mismatch in sampling frequency turns out to be one of the major difficulties in calculating the work done by atmospheric loading. Therefore, in order to obtain more reliable results, our calculation is based on the cross-over points of the orbits. 
The TOPEX/POSEIDON orbit repeat cycle is about 10 days. Since ascending and descending orbits cross in one cycle, there are two samplings at the crossover points during each cycle. Therefore, the sampling frequency at the crossover points is doubled. The increase of sampling gives rise to more accurate results obtained from Eq.(3).

Note that $\eta^{\prime}$ in Eq.(3) is the actual sea level anomaly obtained from the altimeter data, with no barometer correction. Furthermore, $\eta^{\prime}$ used in the calculation is the deviation from the long-term mean sea level, so errors in Geoids should not affect the results of the calculation.

The dataset used in this is from October 3, 1992 to June 2, 2002 (cycle 1-360), which covers $66^{\circ} S-66^{\circ} \mathrm{N}$ with a sample matrix of $127 \times 116$. The zonal resolution is $2.8^{\circ}$, and the meridional resolution is $0.02^{\circ}$ at the northern and southern boundary, and it is $4^{\circ}$ near the equator.

The sea level atmospheric pressure data is taken from the NCEP Reanalysis. This is a daily-mean pressure dataset covering the global with a spatial resolution of $2.5^{\circ} \times 2.5^{\circ}$. From this dataset the time series of $\frac{d p^{\prime}}{d t}$ was calculated and interpolated onto each crossover point in the altimeter dataset.

From the time series of $\frac{d p^{\prime}}{d t}$, the rate of pressure change at each crossover point at any given time can by calculated. Using Eq.(3), mechanical energy input can be calculated accordingly. The horizontal distribution of mechanical energy due to atmospheric loading averaged over the past ten years is shown in Fig. 1, and the global mean rate of energy input is $0.12 \mathrm{~mW} / \mathrm{m}^{2}$, with the global sum of $0.04 T W$. Note that the trajectories of the satellite altimeter do not cover latitudes higher than $66^{\circ}$; thus, our value may underestimate the total energy flux. Previous estimate was 0.01TW by 
Ponte, as cited in Wunsch and Ferrari (2004); however, no detail of his estimate was provided. In comparison with other mechanical energy sources, this seems to be a relatively small contribution; however, the dynamic role of this energy input may be related to the sea level's response to the atmospheric loading, and this needed to be study more carefully.

This energy input is far from being uniformly distributed in space. In fact, it is mostly concentrated around the Southern Oceans and the Storm Tracks in the Northern Hemisphere (Fig. 1). Note that other forms of mechanical energy input from atmosphere to the ocean have a similar pattern, including wind energy input through the geostrophic current, the ageostrophic current (Ekman layer), and the surface waves (Wunsch, 1998; Watanaba and Hibiya, 2002; Alford, 2003; Wang and Huang, 2004a\&b).

Mechanical energy input due to atmospheric loading varies greatly with time because changes in the atmospheric circulation. Thus, it is no surprise that energy input due to atmospheric loading to the world oceans varies slightly from year to year, with amplitude of about 15\% over the past decade (Fig. 2). Note that this is about the same order of the accuracy of the calculation.

\section{Conclusions}

Mechanical energy input due to atmospheric loading is calculated for the world oceans, based on the sea level from the TOPEX/POSEIDON altimeter data on crossover points of the orbits and daily mean sea level pressure from the NCEP reanalysis dataset in the past ten-year period (1993-2002). The rate of total energy input is estimated at 0.04TW, and most energy input is concentrated in the Southern Oceans and the Storm Tracks in the Northern Hemisphere. The present approach may underestimate the 
energy input through atmospheric loading because much of the high frequency events are omitted due to the under-sampling of the sea level signals. Our calculations indicate that energy input from atmospheric loading is a small positive source for the oceanic general circulation. It seems that such a contribution is much smaller than other major sources, such as wind stress and tides, and it may not play important role in the oceanic general circulation; however, further study may needed for potential role of this energy input to the dynamics of oceanic circulation, especially the global response of sea surface elevation to atmospheric pressure perturbations.

Acknowledgment: CLS AVISO OPERATIONS CENTER generously supplied us with the TOPEX/POSEIDON altimeter data. WW and CCQ were supported by the National Nature Science Foundation of China through grant 40476010 and Research Fund for the Doctoral Program of Higher Education through grant 20030423011. RXH was supported by the National Aero-Space Administration through Contract No. 1229833 (NRA-00-OES-05). Woods Hole Contribution \#\#\#\#\#.

\section{References}

Alford, M. H., 2003: Improved global maps and 54-year history of wind-work on the ocean inertial motions. Geophys. Res. Lett., 30(8), 10.1029/2002GL016614.

Fu, L. L. and G. Pihos, 1994: Determining the response of sea level to atmospheric pressure using TOPEX/POSEIDON data. J. Geophys. Res., 99, 24633-24642.

Gaspar, P., and R. M. Ponte, 1997: Relation between sea level and barometric pressure determined from altimeter data and model simulation. J. Geophys. Res., 102, 961-971.

Huang, R. X., 2004. Ocean, energy flow in. Encyclopedia of Energy, C. J. Cleveland (Ed.), Elsevier, Oxford, 497-509.

Wang, W., and R. X. Huang, 2004a: Wind energy input into the surface waves. J. Phys. 
Oceanography., 1276-1280.

Wang, W., and R. X. Huang, 2004b: Wind energy input into the Ekman layer. J. Phys. Oceanography., 1267-1275.

Watanabe, M., and T. Hibiya, 2002: Global estimates of the wind-induced energy flux to inertial motions in the surface mixed layer. Geophys. Res. Lett., 29, 10.1029/2001GL014422.

Wunsch, C., and D. Stammer, 1997: Atmospheric loading and the oceanic "inverted barometer” effect. Rev. Geophys., 35(1), 79-107.

Wunsch, C., 1998: The work done by the wind on the oceanic general circulation, $J$. Phys. Oceanography., 28, 2331-2339.

Wunsch, C. and R. Ferrari, 2004: Vertical mixing, energy, and the general circulation of the oceans. Ann. Rev. of Fluid Mech., 36, 281-314. 

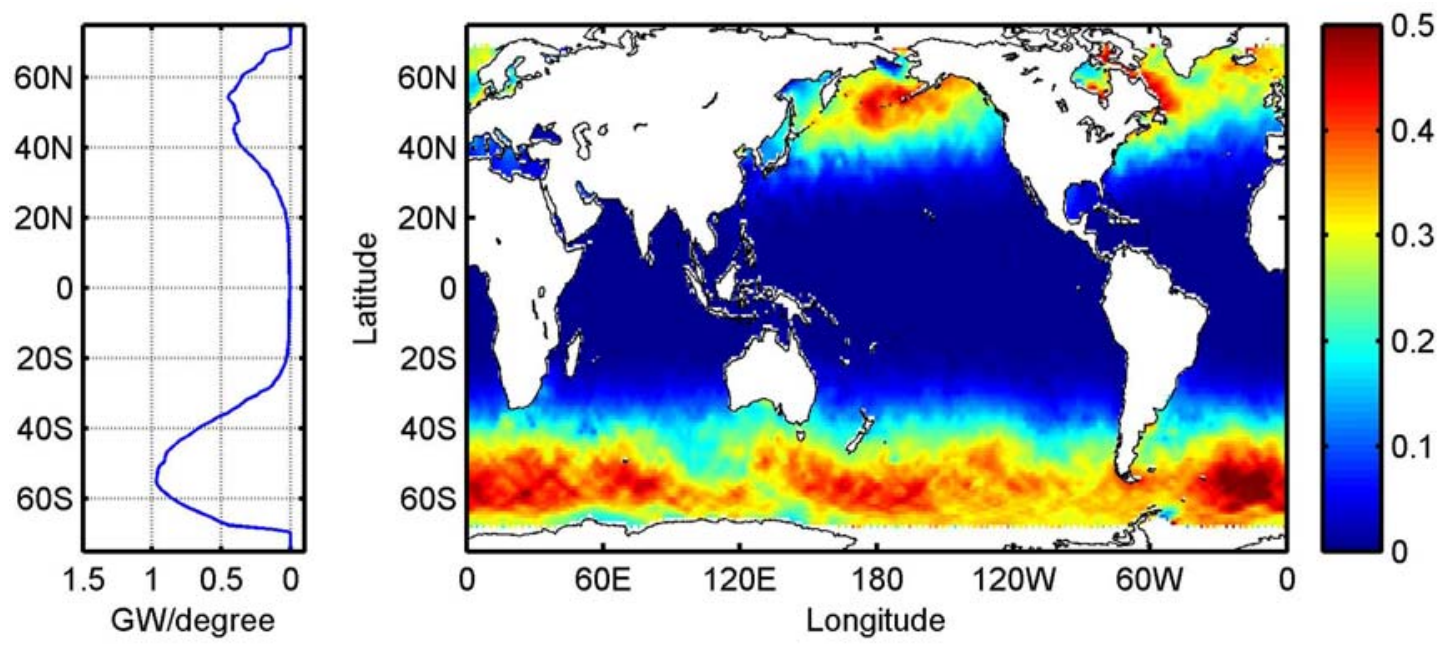

Fig. 1. Global distribution of mechanical energy input due to atmospheric loading ( $m W / m^{2}$ ), averaged over the period of 1993-2002 (right panel) and the zonally integrated mechanical energy (in GW per degree) input due to atmospheric loading (left panel). 


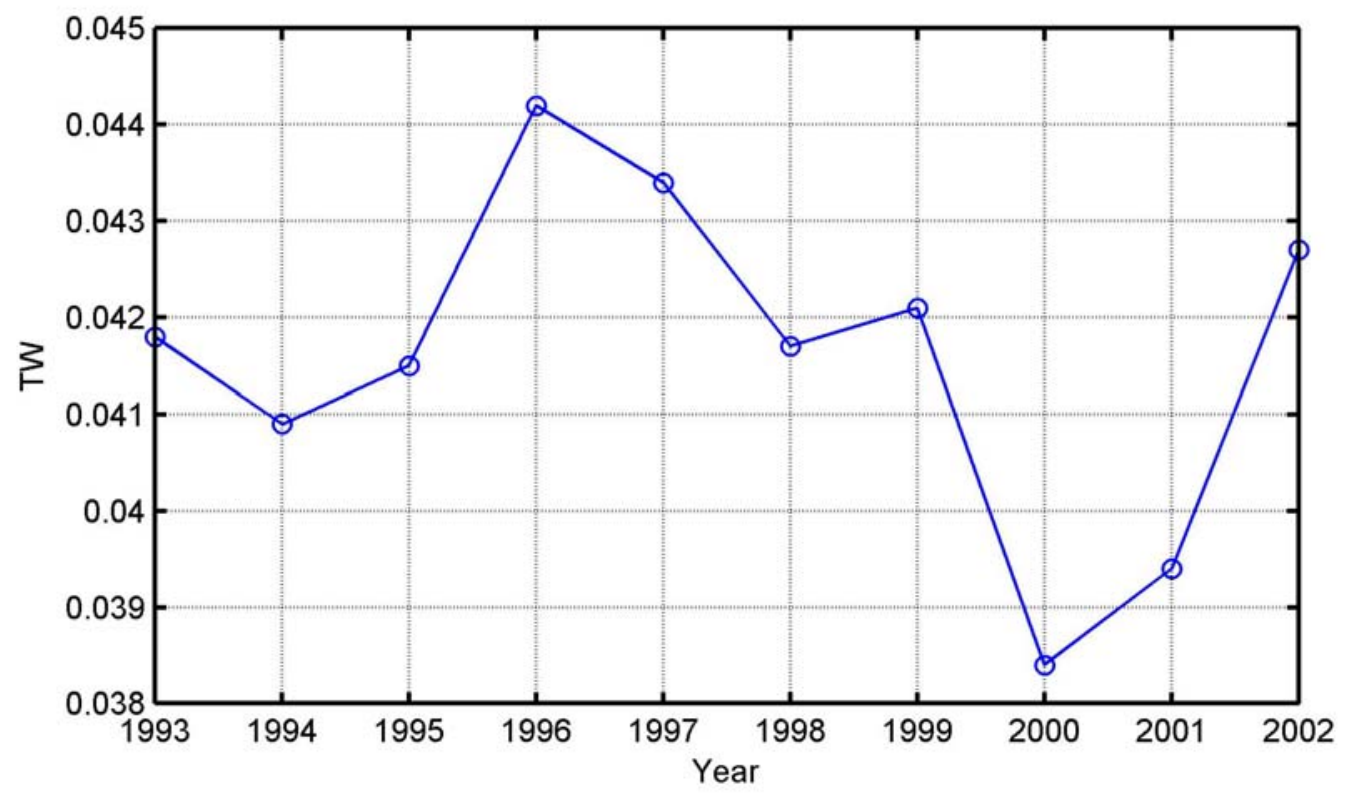

Fig. 2. Interannual variability of the mechanical energy input due to atmospheric loading. 